\title{
Potential Disease-Modifying Effects of Lithium Carbonate in Niemann-Pick Disease, Type C1
}

\begin{abstract}
Shiqian Han ${ }^{1}$, Huiwen Zhang ${ }^{2 *}$, Mengni Yi $^{2}$, Xiaoqing Liu ${ }^{2}$, Gustavo H. B. Maegawa ${ }^{3}$, Yunding Zou ${ }^{4}$, Qijun Wang ${ }^{5,6,7}$, Dianqing $W^{6,7}$ and Zhijia Ye ${ }^{1,8 *}$
\end{abstract}

${ }^{1}$ Department of Tropical Medicine, College of Preventive Medicine, Army Medical University (Third Military Medical University), Chongqing, China, ${ }^{2}$ Department of Pediatric Endocrinology and Genetics, Xinhua Hospital, Shanghai Institute for Pediatric Research, Shanghai Jiao Tong University School of Medicine, Shanghai, China, ${ }^{3}$ Department of Pediatrics, Genetics and Metabolism, University of Florida, Gainesville, FL, United States, ${ }^{4}$ Department of Hematology, Southwest Hospital, Army Medical University (Third Military Medical University), Chongqing, China, ${ }^{5}$ Department of Gastroenterology of Ruijin Hospital, Shanghai Institute of Immunology, Shanghai Jiao Tong University School of Medicine, Shanghai, China, ${ }^{6}$ Departments of Vascular Biology and Therapeutics Program, Yale University School of Medicine, New Haven, CT, United States, ${ }^{7}$ Department of Pharmacology, Yale University School of Medicine, New Haven, CT, United States, ${ }^{8}$ Laboratory Animal Research Center, Chongqing University School of Medicine, Chongqing, China

OPEN ACCESS

Edited by:

Per Svenningsson, Karolinska Institutet (KI), Sweden

Reviewed by:

Martin Paucar,

Karolinska University Hospital,

Sweden

Mark Walterfang,

Royal Melbourne Hospital, Australia

*Correspondence:

Zhiija Ye

yezj918@cqu.edu.cn

Huiwen Zhang

zhanghuiwen@xinhuamed.com.cn

Specialty section:

This article was submitted to

Neuropharmacology,

a section of the journal

Frontiers in Pharmacology

Received: 19 February 2021 Accepted: 25 May 2021 Published: 09 June 2021

Citation:

Han S, Zhang H, Yi M, Liu X, Maegawa GHB, Zou Y, Wang Q, Wu D and Ye Z (2021) Potential DiseaseModifying Effects of Lithium Carbonate in Niemann-Pick Disease, Type C1.

Front. Pharmacol. 12:667361.

doi: 10.3389/fphar.2021.667361
Background: Niemann-Pick disease type C1 (NP-C1) is a rare, autosomal-recessive neurodegenerative disorder with no United States Food and Drug Administration (FDA)approved drug. Lithium has been shown to have considerable neuroprotective effects for neurological disorders such as bipolar disorder, Alzheimer's disease and stroke and has been tested in many clinical trials. However, the pharmacological effect of lithium on NP-C1 neurodegenerative processes has not been investigated. The aim of this study was to provide an initial evaluation of the safety and feasibility of lithium carbonate in patients with NP-C1.

Methods: A total of 13 patients diagnosed with NP-C1 who met the inclusion criteria received lithium orally at doses of 300,600, 900, or 1,200 mg daily. The dose was reduced based on tolerance or safety observations. Plasma 7-ketocholesterol (7-KC), an emerging biomarker of NP-C1, was the primary endpoint. Secondary endpoints included NPC Neurological Severity Scores (NNSS) and safety.

Results: Of the 13 patients with NP-C1 (12-33 years) enrolled, three withdrew (discontinuation of follow-up outpatient visits). The last observed post-treatment values of 7-KC concentrations ( $128 \mathrm{ng} / \mathrm{ml}$, SEM 20) were significantly lower than pretreatment baselines values (185 $\mathrm{ng} / \mathrm{ml}$, SEM 29; $p=0.001$ ). The mean NNSS was improved after lithium treatment at 12 months $(p=0.005)$. Improvement in swallowing capacity was observed in treated patients $(p=0.014)$. No serious adverse events were recorded in the patients receiving lithium.

Conclusion: Lithium is a potential therapeutic option for NP-C1 patients. Larger randomized and double-blind clinical trials are needed to further support this finding.

Clinical Trial Registration: ClinicalTrials.gov, NCT03201627.

Keywords: niemann-pick disease, lithium, clinical trial, 7-ketocholesterol, NNSS 


\section{INTRODUCTION}

Niemann-Pick disease, type C1 (NP-C1) is a rare and progressive neurodegenerative lysosomal disease caused by mutations in NPC1. Cells with a defect in NPC1 lead to the endo/lysosomal accumulation of unesterified cholesterol and glycosphingolipids (Ory, 2000; Vanier, 2010). NP-C1 is a multisystemic inborn lysosomal disorder, and its clinical manifestations include neurological, visceral and psychiatric symptoms and signs (Vanier, 2010). The infantile-onset clinical form of NP-C1 is characterized by visceral symptoms and global neurodevelopmental delay, while late onset forms of NP-C1 with adolescent/adult-onset forms present with a wide spectrum of neurological and psychiatric manifestations (Bonnot et al., 2019). The neurological component of NP-C1 consists mostly of dysarthria, dysphagia, cerebellar ataxia and insidious dementia. The majority of affected patients typically present with vertical supranuclear gaze palsy (Solomon et al., 2005). This progressive neurodegeneration of NP-C1 coupled with delayed diagnosis indicates that manifestation improvement and stabilization and deterioration progression reduction are the priority attainable goals for long-term therapy (Rego et al., 2019).

Miglustat is the only available treatment for NP-C1 patients. Miglustat can stabilize neurological manifestations by inhibiting glycosphingolipid biosynthesis (Patterson et al., 2010; Wraith et al., 2010) and was approved in Europe, Canada, Japan and China but not in the United States. 2-Hydroxypropyl$\beta$-cyclodextrins (HP $\beta C D$ ) has been shown to ameliorate neurological signs and prolong lifespan in both mouse (Liu et al., 2009) and cat (Vite et al., 2015) models of NP-C1. $\mathrm{HP} \beta \mathrm{CD}$ is currently in phase II/III clinical trials. However, the adverse side effects, such as ototoxicity (Crumling et al., 2012; Vite et al., 2015), remain issues that need to be addressed. The emerging approaches for NP-C1 treatment, including arimoclomol (Kirkegaard et al., 2016), histone deacetylase inhibitor therapy (Kim et al., 2007; Alam et al., 2016) and gene therapy (Chandler et al., 2017; Xie et al., 2017; Hughes et al., 2018), have shown significant therapeutic potential in preclinical and clinical studies. Nevertheless, treatment options for NP-C1 are very limited, and new therapies or strategies are needed.

Over the last 2 decades, burgeoning evidence of beneficial neurotrophic and neuroprotective effects of lithium salt has emerged (Rybakowski et al., 2018). Lithium is the first-line mood stabilizer for the treatment of bipolar disorder (McIntyre et al., 2020). Lithium was also demonstrated to improve phenotypes in animal models of clinical neurodegenerative conditions, including Alzheimer's disease (Fiorentini et al., 2010; Leroy et al., 2010; Zhang et al., 2011), Huntington's disease (Wei et al., 2001; Senatorov et al., 2004), Parkinson's disease (Youdim and Arraf, 2004; Qi et al., 2017), and stroke (Doeppner et al., 2017). Recently, hyperphosphorylated tau was observed in NP-C1 postmortem brains and in patientderived neurons (Burbulla et al., 2021). Lithium, a selective inhibitor of glycogen synthase kinase $3 \beta$ (GSK3 $\beta$ ), could significantly decrease hyperphosphorylation of tau (Madra and Sturley, 2010). Moreover, the combination of bipolar medications, including lithium, was reported to increase the expression of NPC1 in a human neuronal cell line (Kidnapillai et al., 2019). The preceding study provided a promising way to investigate lithium as a treatment for NP-C1 patients. In this study, we conducted an open-label, nonrandomized and singlearm study to provide an initial evaluation of the safety and potential efficacy of lithium in patients with NP-C1.

\section{METHODS}

\section{Participants}

A pilot open-label, nonrandomized, single-arm study was performed to assess the safety and clinical efficacy of oral lithium carbonate in patients with NP-C1 (ClinicalTrials.gov Identifier: NCT03201627) from July 2017 to November 2019. All patients were recruited at Xinhua Hospital, Shanghai Jiao Tong University School of Medicine, China. Patients with NP$\mathrm{C} 1$ were strictly selected by the inclusion and exclusion criteria (Supplementary Table S1). Eligible patients with two mutant NPC1 alleles had neurological manifestations, were aged 7 years or older, and were required to participate in all aspects of the clinical study. All patients or their legal guardians gave written informed consent prior to their participation in the study.

\section{Assignment and Procedures}

Patients were sequentially assigned to receive a starting dose of $300 \mathrm{mg}$ or $600 \mathrm{mg}$ oral lithium carbonate pills with slow release. The lithium blood content was assessed one week after taking carbonate lithium for full clinical efficacy. Then patients had an outpatient visit once per month or every three months and received four phone calls every month during the treatment period. Any dose adjustment was made through a comprehensive decision combined with treatment efficacy and side effects. The NPC Neurological Severity Score (NNSS) were assessed at baseline and then approximately every three months or every 6 months. The NNSS is a Likertlike scale that assesses the neurological progression of NP-C1 in nine major and eight minor domains (Yanjanin et al., 2010; Ory et al., 2017).

\section{Outcomes}

The primary outcome was the change of 7-Ketocholesterol (7$\mathrm{KC)}$ concentration in plasma after drug administration compared with the 7-KC values before oral lithium carbonate administration. The quantification analysis of plasma $7-\mathrm{KC}$ was carried out with LC/MS-MS by following the protocol we previously established (Lin et al., 2014; Zhang et al., 2014). The plasma lithium value was measured by IC-MS. Blood samples were obtained at fasting state before intake of morning medication (trough level).

The scoring of NNSS subdomains was performed based on the procedure recommended by the NIH (Ory et al., 2017). Two doctors who had more than 5 years of experience in NP-C1 diagnosis independently performed the NNSS assessments. The score was directly selected if the same score was given by two 


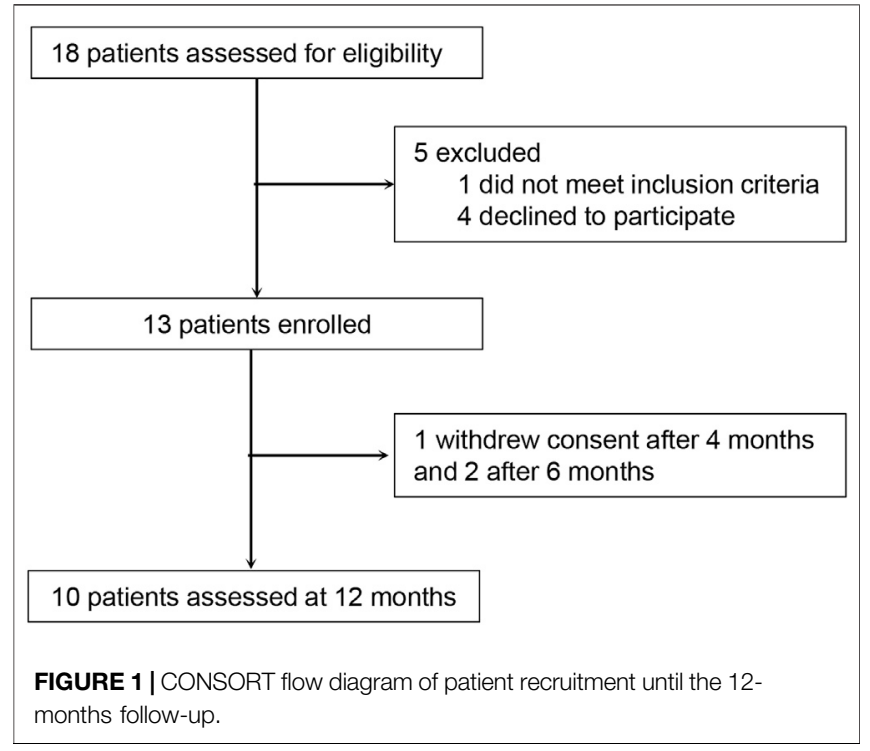

doctors, while the scoring was determined by discussion if different scores were given by two doctors.

The severity of adverse events was graded according to the Common Terminology Criteria for Adverse Events (CTCAE), Version 4.0.

\section{Statistical Analysis}

The human study was a pre- and posttreatment design in which the baseline of a patient served as his/her own control. Patient characteristics were summarized using descriptive statistics. A paired $t$-test was used to assess changes in 7-KC concentrations. A nonparametric method, the Wilcoxon signed rank test, was used for the primary analysis for efficacy and the change in each subscale. Spearman correlation analysis was conducted to examine the association of some baseline characteristics and lithium concentration with changes in NNSS. Pearson's correlation coefficient was used to assess the 7-KC data correlations.

Statistical analyses were performed with GraphPad Prism software (version 8.0.1). The statistical significance was set as $p<0.05$, two-sided.

\section{RESULTS}

\section{Baseline Characteristics of the Patients}

A total of 18 patients with NP-C1 aged 12 years or older were recruited for this single-arm pilot clinical study to assess the safety and clinical efficacy of oral lithium carbonate (Figure 1). Five patients were excluded due to an inability to comply with the study procedures (four patients) or a failure to meet the inclusion criteria (one patient). 13 patients were eligible and were enrolled in the study. Two patients were followed up only for 6 months and one patient was able to complete the 4-month assessment due to the discontinuation of follow-up outpatient visits. The remaining 10 patients were followed up for 12 months. The baseline characteristics are provided in Table $\mathbf{1}$ and
TABLE 1 | Baseline characteristics of patients.

\begin{tabular}{lc}
\hline Characteristic & $\begin{array}{c}\text { Total } \\
\text { patients }(\boldsymbol{n}=\mathbf{1 3})\end{array}$ \\
\hline $\begin{array}{l}\text { Age at baseline (years) } \\
\text { Mean (SD) }\end{array}$ & $19(6.65)$ \\
Range & $12-33$ \\
Sex & \\
Male (\%) & $7(54 \%)$ \\
Female (\%) & $6(46 \%)$ \\
Age of first neurological symptom, years & \\
Mean (SD) & $9.62(2.47)$ \\
Range & $7-13$ \\
Age of diagnosis, years & \\
Mean (SD) & $18.15(6.57)$ \\
Range & $10-31$ \\
Miglustat use, $\mathbf{n}$ (\%) & \\
Yes & $2(15 \%)$ \\
No & $11(85 \%)$
\end{tabular}

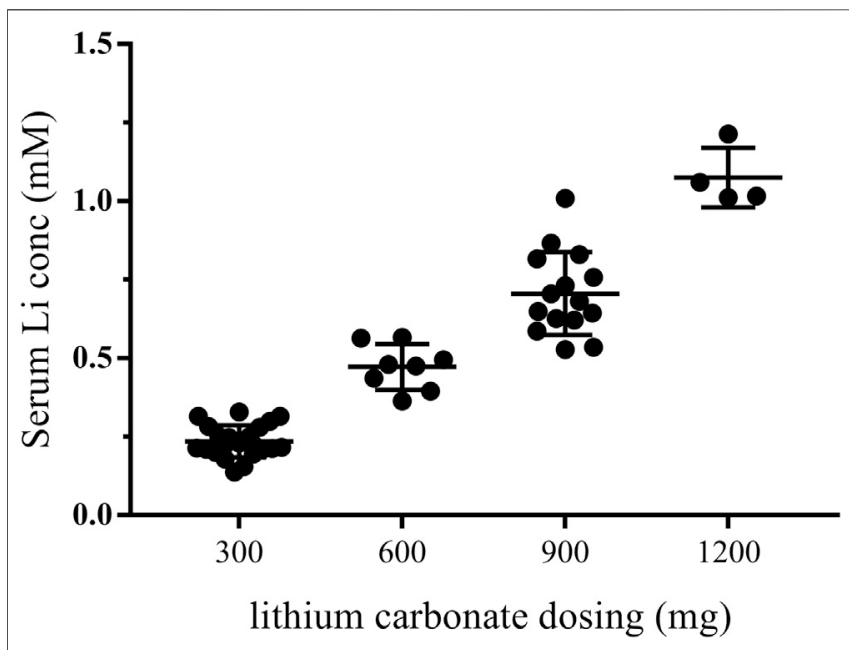

FIGURE 2 | Plasma lithium levels after receiving lithium carbonate. Every point represents that plasma lithium levels were detected after receiving lithium at doses of $300,600,900$, or 1,200 $\mathrm{mg}$. The first month was the dose adjustment period. and plasma lithium levels were measured by IC-MS once a week. The plasma lithium levels were then examined approximately every 3 months after the first month. Therefore, the lithium plasma levels of one patient were detected several times at one dose. conc, concentration.

Supplementary Table S2. We used the last follow-up data as the endpoint for these 13 patients who took lithium carbonate.

During the first month of the treatment (the dose adjustment period), the patients were given lithium doses ranging from 300 to $900 \mathrm{mg} /$ day, and serum lithium levels were measured at least once a week. Detailed lithium dosing information is provided in Supplementary Table S3. The plasma lithium levels were then examined approximately every 3 months after the first month, and the participant's plasma lithium concentrations were positively associated with oral doses of lithium carbonate (Figure 2). Because the initial goal was to reach the upper end of the therapeutic lithium concentrations range, i.e., between 0.6 and 
A

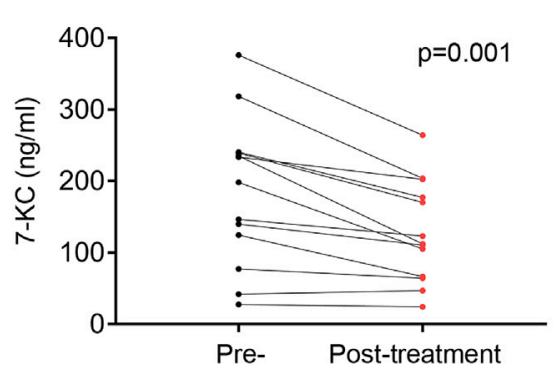

$7-\mathrm{KC}$ at baseline $(\mathrm{ng} / \mathrm{ml})$

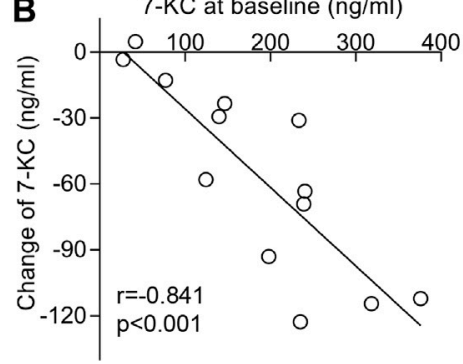

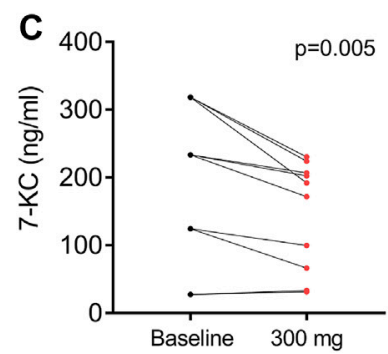
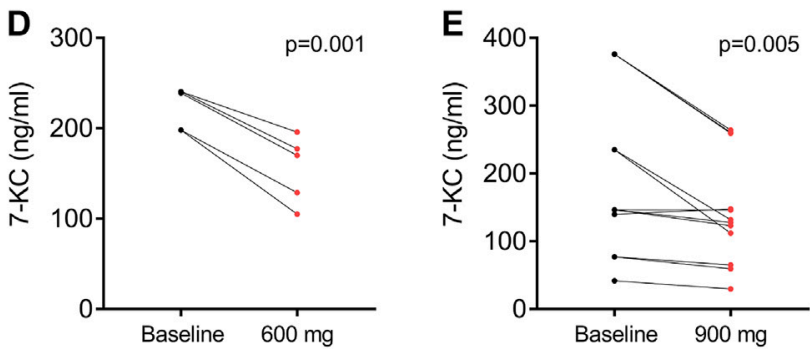

FIGURE 3 | Effect of lithium on 7-KC. (A) Plasma 7-KC concentration measured before and at the last follow-up after lithium administration in 13 patients. (B) The association of plasma 7-KC at baseline with changes in 7-KC. Concentration change of plasma 7-KC after oral doses of $300 \mathrm{mg}$ (C), $600 \mathrm{mg}$ (D) or $900 \mathrm{mg}$ (E) of lithium carbonate. Each paired set of pre- and post-values represent an individual dose: 10 doses among four patients for $300 \mathrm{mg}$, five doses among three patients for $600 \mathrm{mg}$ and 12 doses among six patients for $900 \mathrm{mg} r$ is the Pearson correlation coefficient.

$1.0 \mathrm{mM}$, which is expected under treatment of bipolar disorder (Hopkins and Gelenberg, 2000), some patients received doses as high as $1,200 \mathrm{mg} /$ day. The reduction of the lithium dose was guided by safety and tolerance observations.

\section{Reduction in 7-KC}

In our previous research, we reported that the cholesterol oxidation product 7-KC was detected at elevated levels in NP-C1 patients (Lin et al., 2014; Zhang et al., 2014), and that 7-KC could serve as a specific biomarker for the disease (Porter et al., 2010; Stampfer et al., 2013). This study included measurements of plasma 7-KC concentrations before and after lithium study administration. The last observed posttreatment values of 7-KC concentrations (128 ng/ml, SEM 20) were significantly decreased compared with the pretreatment baseline values (185 ng/ml, SEM 29; $p=0.001$ ) (Figure 3A). Importantly, higher baseline 7-KC concentrations were significantly correlated with greater reductions in the 7-KC concentration (Figure 3B; $r=$ $-0.841 ; p<0.001)$. We also found that $7-\mathrm{KC}$ significantly decreased after oral administration of 300, 600 and $900 \mathrm{mg}$ lithium carbonate tablets (Figures 3C-E), but plasma lithium concentration was not significantly correlated with the change in $7-\mathrm{KC}$ concentration (Supplementary Figure S1, $r=0.028 ; p=0.928$ ).

\section{NPC Neurological Severity Score}

Clinical benefit was ascertained by comparing the total NNSS before lithium treatment with that after lithium treatment. Ten patients were assessed at 12 months. All 10 patients achieved reductions in their NNSS after 12-months, with an average 2.6-point decrease ( $p=$ 0.005) (Table 2). Among the 17 NNSS domains examined, the reduction in the swallowing domain score was statistically significant $(p=0.014)$. There were trends of reduction in incontinence scores $(p=0.083)$ (Table 2). The examination of the NNSS at the interim time points in these 13 lithium-treated patients revealed no substantial deleterious effect of lithium (Figure 4, Supplementary Table S4). The decrease in the total NNSS was mainly contributed by improvement in the swallowing domain score, assessing the level of dysphagia (Figures 5A,B). Natural history studies have shown that swallowing ability is gradually impaired from the time of disease onset (Wraith et al., 2009). Together, these results suggest that lithium may be an available therapeutic option for NP-C1 patients. Other characteristics, including sex, age at diagnosis, the age of onset, length of treatment duration (data not shown), and lithium concentration, failed to significantly correlate with the reduction of scores in the NNSS (Supplementary Figure S2, $r=$ $-0.219 ; p=0.472$ ).

\section{Safety Evaluation}

Lithium treatment was generally well tolerated in NP-C1 patients. Adverse events, including hypothyroidism (3 patients) and benign leukocytosis (5 patients), as well as rare occurrences of manic episodes ( 2 patients) and drooling (1 patient), were observed at high doses (Table 3 ).

\section{DISCUSSION}

Lithium is an agent that has been in use for the treatment of psychiatric disorders for decades after research by John Cade, an Australian psychiatrist (Cade, 1949). To our knowledge, this is the first open label study to assess the safety and potential efficacy 
TABLE 2 | Comparison of NNSS subscales before and after 12 months of lithium treatment in 10 NP-C1 patients.

\begin{tabular}{|c|c|c|c|}
\hline & Prior treatment (SD) & After treatment (SD) & $p$ value \\
\hline Eye movement & $2.7(0.5)$ & $2.7(0.5)$ & - \\
\hline Ambulation & $2.2(0.6)$ & $2.0(0)$ & 0.317 \\
\hline Speech & $1.4(0.7)$ & $1.3(0.7)$ & 0.317 \\
\hline Swallowing & $1.8(0.9)$ & $0.9(0.7)$ & 0.014 \\
\hline Fine motor & $2.1(0.7)$ & $2.0(0.8)$ & 0.317 \\
\hline Cognition & $3.6(1.0)$ & $3.4(1.0)$ & 0.157 \\
\hline Hearing & $0.7(1.3)$ & $0.6(1.1)$ & 0.317 \\
\hline Memory & $1.7(0.8)$ & $1.7(0.8)$ & - \\
\hline Seizures & $0.6(1.3)$ & $0.5(1.1)$ & 0.317 \\
\hline Gelastic cataplexy & $0.4(0.8)$ & $0.2(0.4)$ & 0.157 \\
\hline Hyperreflexia & $1.2(0.6)$ & $1.1(0.7)$ & 0.317 \\
\hline Narcolepsy & $0(0)$ & $0(0)$ & - \\
\hline Incontinence & $0.6(0.7)$ & $0.3(0.7)$ & 0.083 \\
\hline Behavior & $0(0)$ & $0(0)$ & - \\
\hline Auditory brain response (ABR) & $1.0(0)$ & $1.0(0)$ & - \\
\hline Psychiatric & $0.3(0.7)$ & $0(0)$ & 0.18 \\
\hline Respiratory & $0(0)$ & $0(0)$ & - \\
\hline Total score & $20.3(5.4)$ & $17.7(4.9)$ & 0.005 \\
\hline
\end{tabular}

The bold values mean $p<0.05$.
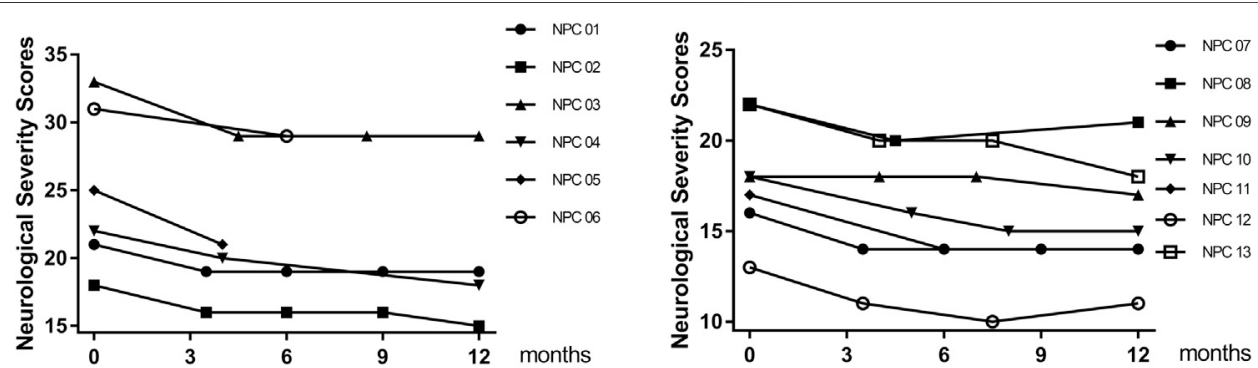

FIGURE 4 | Effect of lithium on NNSS. The total NNSS of 13 patients who accepted lithium treatment obtained at multiple time points within 12 months.

of lithium treatment in patients with NP-C1. Our results preliminarily demonstrated that lithium carbonate tablets may have the potential to ameliorate the neurological phenotypes of NP-C1 patients. Lithium treatment significantly improved overall NNSS scores and swallowing subdomain scores. Furthermore, lithium significantly reduced plasma $7-\mathrm{KC}$ in patients with NP$\mathrm{C} 1$. We also demonstrated that lithium was well tolerated in patients with NP-C1. The lower incidence of side effects in this study was due to the low doses of lithium used in the NP-C1 patients.

Dysphagia was present in 55-80\% of NP-C1 patients and was identified as one of the greatest risk factors for NP-C1 (Walterfang et al., 2012). Dysphagia is a main cause of aspiration pneumonia, contributing to two-thirds of deaths in NP-C1 patients (Petroianni et al., 2006; Walterfang et al., 2012). The improvement in swallowing capacity was closely related to the quality of life of NP-C1 patients. A recent study demonstrated that the determination of swallowing capacity could be considered as a clinically functional outcome indicator for future therapeutic interventions in NP-C1 (Solomon et al., 2020). In our study, most NP-C1 patients suffered from dysphagia during the disease course. Lithium carbonate significantly reduced the swallowing subdomain scores, indicating that lithium treatment may improve swallowing capacity and quality of life in NP-C1 patients. Cerebellar ataxia is one of the most specific manifestations in NP-C1 patients, and it correlates with gait ataxia, dysarthria, dysphagia and swallowing (Schmahmann, 2004). In preliminary studies, we demonstrated that lithium carbonate treatment significantly protected cerebellar Purkinje cells, improved food intake and extended survival in vivo studies using $\mathrm{NP}-\mathrm{Cl}^{-/-}$and $\mathrm{NP}-\mathrm{Cl}^{I 1061 T}$ mouse models (unpublished data). Therefore, we speculate that lithium may improve swallowing difficulties by protecting Purkinje cell in the NP-C1 patients. Moreover, in a 48-weeks phase 2 trial of the efficacy of lithium in patients with spinocerebellar ataxia type 3 (an adult-onset disorder featured by cerebellar ataxia), ataxic manifestations at endpoint were significantly delayed in the treated group compared to the placebo group, and it was suggested that lithium could be beneficial to patients at a relatively early stage of the disease and to specific symptoms, for example cerebellar dysfunction (Saute et al., 2014).

Our biomarker data also support the clinical efficacy of lithium. The plasma content of oxysterol chemicals, including 

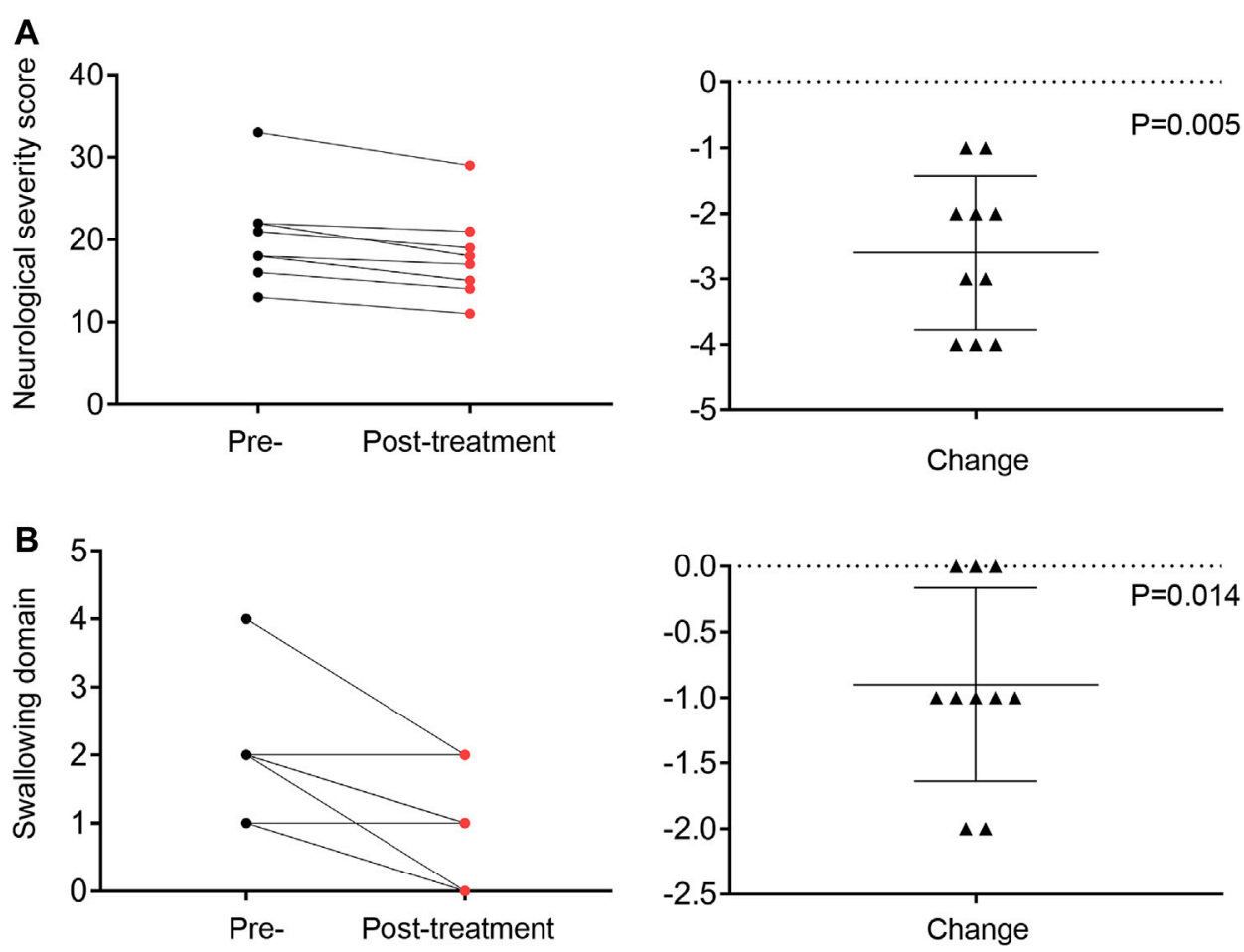

FIGURE 5 | The total NNSS (A) and swallowing (B) domain score recorded at the pre-study and 12-months visits. Change in total score and swallowing domain score from the pre-study to the final visit assessment.

TABLE 3 | Selected adverse events.

Event-no. (\%)

Mania episode Increased drooling

Benign leukocytosis

Transient hypothyroidism

7-KC, has been shown to be highly sensitive and specific in the detection of NP-C1 (Lin et al., 2014; Zhang et al., 2014). The plasma levels of 7-KC have significant correlations with different levels of disease severity of NP-C1 patients (Porter et al., 2010; Stampfer et al., 2013), and the decrease of 7-KC was beneficial to NPC1 mutant feline model after treated by HP $\beta C D$ (Porter et al., 2010). Our data showed that plasma $7-K C$ was significantly reduced after oral doses of 300, 600 and $900 \mathrm{mg}$ of lithium. In addition, higher baseline levels of plasma $7-\mathrm{KC}$ were significantly correlated with greater reductions in patients after lithium administration. These findings raise the possibility that lithium may ameliorate the clinical NP-C1 severity. The mechanism underlying the effect of lithium on $7-\mathrm{KC}$ is not clear. Lithium has been shown to attenuate DNA damage, mitochondrial dysfunction and lipid peroxidation by inhibiting GSK-3 $\beta$ and activating NRF-2 antioxidant response (Khairova et al., 2012; Castillo-Quan et al., 2016; Machado-Vieira, 2018). Lithium may lower 7-KC by modulating oxidative stress and lipid metabolism.
The interpretation of the changes in the NNSS results is limited in the context of a pilot study without placebo control. Considering the small sample size of this study, these potential effects of lithium definitely need more investigation in a larger randomized study. From a safety standpoint, no substantial deleterious effect of lithium was observed, including any adverse effect on motor and hearing features. Moreover, compared with other treatments for NP-C1, the advantages of lithium are readily available and relatively inexpensive.

We also explored oral doses of lithium between 300 and $1,200 \mathrm{mg}$ per month and found no significant impact of average plasma lithium levels on the change in 7-KC levels and NNSS (Supplementary Figures S1, S2). A possible reason for this lack of correlation may be that the lithium dose even at $300 \mathrm{mg} /$ day reached the plateau of the dose-response range. In future large studies, $300 \mathrm{mg} /$ day may be considered the starting point.

In conclusion, we carried out an open-label pilot study to assess the safety, feasibility and tolerability of lithium carbonate in NP-C1 patients over a 12-month period. Lithium carbonate was safe and well tolerated and showed potential therapeutic effects on plasma 7 -KC level reductions and swallowing improvement in NP-C1 patients. Notably, combination therapies of lithium with miglustat or $\mathrm{HP} \beta C D$ may have more potent therapeutic effects. Future prospective, randomized clinical trials with large sample sizes are needed to confirm this finding. 


\section{DATA AVAILABILITY STATEMENT}

The original contributions presented in the study are included in the article/Supplementary Material, further inquiries can be directed to the corresponding authors.

\section{ETHICS STATEMENT}

The studies involving human participants were reviewed and approved by Applicable Institutional Review Board of Xinhua Hospital, Shanghai Jiao Tong University School of Medicine, China. Written informed consent to participate in this study was provided by the participants' legal guardian/next of kin. Written informed consent was obtained from the individual(s), and minor(s)' legal guardian/next of kin, for the publication of any potentially identifiable images or data included in this article.

\section{AUTHOR CONTRIBUTIONS}

$\mathrm{SH}$ and HZ contributed to the conception of the study, collection and analysis of data, preparing the figures and drafting the manuscript; GM contributed to the analysis and interpretation of data and reviewing the manuscript; $\mathrm{MY}$ and $\mathrm{XL}$ contributed to acquisition, analysis and interpretation of data; YZ, QW and DW analyzed and discussed the manuscript; ZY designed and

\section{REFERENCES}

Alam, M. S., Getz, M., and Haldar, K. (2016). Chronic Administration of an HDAC Inhibitor Treats Both Neurological and Systemic Niemann-Pick Type C Disease in a Mouse Model. Sci. Transl. Med. 8, 326ra23. doi:10.1126/ scitranslmed.aad 9407

Bonnot, O., Klünemann, H.-H., Velten, C., Torres Martin, J. V., and Walterfang, M. (2019). Systematic Review of Psychiatric Signs in Niemann-Pick Disease Type C. World J. Biol. Psychiatry 20, 320-332. doi:10.1080/15622975.2018.1441548 Burbulla, L. F., Mc Donald, J. M., Valdez, C., Gao, F., Bigio, E. H., and Krainc, D. (2021). Modeling Brain Pathology of Niemann-Pick Disease Type C Using Patient-Derived Neurons. Mov Disord. 36, 1022-1027. doi:10.1002/mds.28463

Cade, J. F. (1949). Lithium Salts in the Treatment of Psychotic Excitement. Med. J. Australia.

Castillo-Quan, J. I., Li, L., Kinghorn, K. J., Ivanov, D. K., Tain, L. S., Slack, C., et al. (2016). Lithium Promotes Longevity through GSK3/NRF2-dependent Hormesis. Cel Rep. 15, 638-650. doi:10.1016/j.celrep.2016.03.041

Chandler, R. J., Williams, I. M., Gibson, A. L., Davidson, C. D., Incao, A. A., Hubbard, B. T., et al. (2017). Systemic AAV9 Gene Therapy Improves the Lifespan of Mice with Niemann-Pick Disease, Type C1. Hum. Mol. Genet. 26, ddw367-64. doi:10.1093/hmg/ddw367

Crumling, M. A., Liu, L., Thomas, P. V., Benson, J., Kanicki, A., Kabara, L., et al. (2012). Hearing Loss and Hair Cell Death in Mice Given the CholesterolChelating Agent Hydroxypropyl- $\beta$-Cyclodextrin. PLoS One 7, e53280. doi:10. 1371/journal.pone.0053280

Doeppner, T. R., Kaltwasser, B., Sanchez-Mendoza, E. H., Caglayan, A. B., Bähr, M., and Hermann, D. M. (2017). Lithium-induced Neuroprotection in Stroke Involves Increased miR-124 Expression, Reduced RE1-Silencing Transcription Factor Abundance and Decreased Protein Deubiquitination by GSK3 $\beta$ Inhibition-independent Pathways. J. Cereb. Blood Flow Metab. 37, 914-926. doi:10.1177/0271678X16647738

Fiorentini, A., Rosi, M. C., Grossi, C., Luccarini, I., and Casamenti, F. (2010). Lithium Improves Hippocampal Neurogenesis, Neuropathology and Cognitive supervised the study, analyzed the data, wrote and revised the manuscript.

\section{FUNDING}

The study was funded by Shanghai Science and Technology Committee (19140904100), Shanghai Municipal Education Commission-Gaofeng Clinical Medicine Grant Support (20152520), the National Natural Science Foundation (81570516, 31371393), and Emphasis Basic Medical Scientific Research Project Fund of China (AWS17J007).

\section{ACKNOWLEDGMENTS}

We thank the patients with NP-C1 and their families for participation in this study. We also thank Wenjuan Jiang, Feng $\mathrm{Xu}$ and Junxia Liu for providing assistance in clinical data collection.

\section{SUPPLEMENTARY MATERIAL}

The Supplementary Material for this article can be found online at: https://www.frontiersin.org/articles/10.3389/fphar.2021.667361/ full\#supplementary-material

Functions in APP Mutant Mice. PLoS One 5, e14382. doi:10.1371/journal.pone. 0014382

Hopkins, H. S., and Gelenberg, A. J. (2000). Serum Lithium Levels and the Outcome of Maintenance Therapy of Bipolar Disorder. Bipolar Disord. 2, 174-179. doi:10.1034/j.1399-5618.2000.020304.x

Hughes, M. P., Smith, D. A., Morris, L., Fletcher, C., Colaco, A., Huebecker, M., et al. (2018). AAV9 Intracerebroventricular Gene Therapy Improves Lifespan, Locomotor Function and Pathology in a Mouse Model of Niemann-Pick Type C1 Disease. Hum. Mol. Genet. 27, 3079-3098. doi:10.1093/hmg/ddy212

Khairova, R., Pawar, R., Salvadore, G., Juruena, M. F., de Sousa, R. T., Soeiro-deSouza, M. G., et al.(2012). Effects of Lithium on Oxidative Stress Parameters in Healthy Subjects. Mol. Med. Rep. 5, 680-682. doi:10.3892/mmr.2011.732

Kidnapillai, S., Bortolasci, C. C., Panizzutti, B., Spolding, B., Connor, T., Bonifacio, K., et al. (2019). Drugs Used in the Treatment of Bipolar Disorder and Their Effects on Cholesterol Biosynthesis - A Possible Therapeutic Mechanism. World J. Biol. Psychiatry 20, 766-777. doi:10.1080/15622975.2019.1669823

Kim, S.-J., Lee, B.-H., Lee, Y.-S., and Kang, K.-S. (2007). Defective Cholesterol Traffic and Neuronal Differentiation in Neural Stem Cells of Niemann-Pick Type C Disease Improved by Valproic Acid, a Histone Deacetylase Inhibitor. Biochem. Biophysical Res. Commun. 360, 593-599. doi:10.1016/j.bbrc.2007.06.116

Kirkegaard, T., Gray, J., Priestman, D. A., Wallom, K.-L., Atkins, J., Olsen, O. D., et al. (2016). Heat Shock Protein-Based Therapy as a Potential Candidate for Treating the Sphingolipidoses. Sci. Transl. Med. 8, 355ra118. doi:10.1126/ scitranslmed.aad9823

Leroy, K., Ando, K., Héraud, C., Yilmaz, Z., Authelet, M., Boeynaems, J.-M., et al. (2010). Lithium Treatment Arrests the Development of Neurofibrillary Tangles in Mutant Tau Transgenic Mice with Advanced Neurofibrillary Pathology. Jad 19, 705-719. doi:10.3233/JAD-2010-1276

Lin, N., Zhang, H., Qiu, W., Ye, J., Han, L., Wang, Y., et al. (2014). Determination of 7-ketocholesterol in Plasma by LC-MS for Rapid Diagnosis of Acid SMaseDeficient Niemann-Pick Disease. J. Lipid Res. 55, 338-343. doi:10.1194/jlr. D044024

Liu, B., Turley, S. D., Burns, D. K., Miller, A. M., Repa, J. J., and Dietschy, J. M. (2009). Reversal of Defective Lysosomal Transport in NPC Disease Ameliorates 
Liver Dysfunction and Neurodegeneration in the Npc1-/- Mouse. Proc. Natl. Acad. Sci. 106, 2377-2382. doi:10.1073/pnas.0810895106

Machado-Vieira, R. (2018). Lithium, Stress, and Resilience in Bipolar Disorder: Deciphering This Key Homeostatic Synaptic Plasticity Regulator. J. Affective Disord. 233, 92-99. doi:10.1016/j.jad.2017.12.026

Madra, M., and Sturley, S. L. (2010). Niemann-Pick Type C Pathogenesis and Treatment: from Statins to Sugars. Clin. Lipidol. 5, 387-395. doi:10.2217/clp. 10.19

McIntyre, R. S., Berk, M., Brietzke, E., Goldstein, B. I., López-Jaramillo, C., Kessing, L. V., et al. (2020). Bipolar Disorders. The Lancet 396, 1841-1856. doi:10.1016/ S0140-6736(20)31544-0

Ory, D. (2000). Niemann-Pick Type C: a Disorder of Cellular Cholesterol Trafficking. Biochim. Biophys. Acta 1529, 331-339. doi:10.1016/s13881981(00)00158-x

Ory, D. S., Ottinger, E. A., Farhat, N. Y., King, K. A., Jiang, X., Weissfeld, L., et al. (2017). Intrathecal 2-Hydroxypropyl- $\beta$-Cyclodextrin Decreases Neurological Disease Progression in Niemann-Pick Disease, Type C1: a Non-randomised, Open-Label, Phase 1-2 Trial. The Lancet 390, 1758-1768. doi:10.1016/S01406736(17)31465-4

Patterson, M. C., Vecchio, D., Jacklin, E., Abel, L., Chadha-Boreham, H., Luzy, C., et al. (2010). Long-term Miglustat Therapy in Children with Niemann-Pick Disease Type C. J. Child. Neurol. 25, 300-305. doi:10.1177/0883073809344222

Petroianni, A., Ceccarelli, D., Conti, V., and Terzano, C. (2006). Aspiration Pneumonia. Pathophysiological Aspects, Prevention and Management. A Review. Panminerva Med. 48, 231-239.

Porter, F. D., Scherrer, D. E., Lanier, M. H., Langmade, S. J., Molugu, V., Gale, S. E., et al. (2010). Cholesterol Oxidation Products Are Sensitive and Specific BloodBased Biomarkers for Niemann-Pick C1 Disease. Sci. Translational Med. 2, 56ra81. doi:10.1126/scitranslmed.3001417

Qi, L., Tang, Y., He, W., Pan, H., Jiang, W., Wang, L., et al. (2017). Lithium Chloride Promotes Neuronal Differentiation of Rat Neural Stem Cells and Enhances Neural Regeneration in Parkinson's Disease Model. Cytotechnology 69, 277-287. doi:10.1007/s10616-016-0056-1

Rego, T., Farrand, S., Goh, A. M. Y., Eratne, D., Kelso, W., Mangelsdorf, S., et al. (2019). Psychiatric and Cognitive Symptoms Associated with Niemann-Pick Type C Disease: Neurobiology and Management. CNS Drugs 33, 125-142. doi:10.1007/s40263-018-0599-0

Rybakowski, J., Suwalska, A., and Hajek, T. (2018). Clinical Perspectives of Lithium's Neuroprotective Effect. Pharmacopsychiatry 51, 194-199. doi:10. 1055/s-0043-124436

Saute, J. A. M., de Castilhos, R. M., Monte, T. L., Schumacher-Schuh, A. F., Donis, K. C., D'Ávila, R., et al. (2014). A Randomized, Phase 2 Clinical Trial of Lithium Carbonate in Machado-Joseph Disease. Mov Disord. 29, 568-573. doi:10.1002/mds.25803

Schmahmann, J. D. (2004). Disorders of the Cerebellum: Ataxia, Dysmetria of Thought, and the Cerebellar Cognitive Affective Syndrome. Jnp 16, 367-378. doi:10.1176/jnp.16.3.367

Senatorov, V. V., Ren, M., Kanai, H., Wei, H., and Chuang, D.-M. (2004). Shortterm Lithium Treatment Promotes Neuronal Survival and Proliferation in Rat Striatum Infused with Quinolinic Acid, an Excitotoxic Model of Huntington's Disease. Mol. Psychiatry 9, 371-385. doi:10.1038/sj.mp.4001463

Solomon, B. I., Smith, A. C., Sinaii, N., Farhat, N., King, M. C., Machielse, L., et al. (2020). Association of Miglustat with Swallowing Outcomes in Niemann-Pick Disease, Type C1. JAMA Neurol. 77, 1564-1566. doi:10.1001/jamaneurol.2020.3241

Solomon, D., Winkelman, A. C., Zee, D. S., Gray, L., and Büttner-ennever, J. (2005). Niemann-Pick Type C Disease in Two Affected sisters: Ocular Motor Recordings and Brain-Stem Neuropathology. Ann. N. Y Acad. Sci. 1039, 436-445. doi:10.1196/annals.1325.041
Stampfer, M., Theiss, S., Amraoui, Y., Jiang, X., Keller, S., Ory, D. S., et al. (2013) Niemann-Pick Disease Type C Clinical Database: Cognitive and Coordination Deficits Are Early Disease Indicators. Orphanet J. Rare Dis. 8, 35. doi:10.1186/ 1750-1172-8-35

Vanier, M. T. (2010). Niemann-Pick Disease Type C. Orphanet J. Rare Dis. 5, 16. doi:10.1186/1750-1172-5-16

Vite, C. H., Bagel, J. H., Swain, G. P., Prociuk, M., Sikora, T. U., Stein, V. M., et al. (2015). Intracisternal Cyclodextrin Prevents Cerebellar Dysfunction and Purkinje Cell Death in Feline Niemann-Pick Type C1 Disease. Sci. Transl. Med. 7, 276ra26. doi:10.1126/scitranslmed.3010101

Walterfang, M., Chien, Y.-H., Imrie, J., Rushton, D., Schubiger, D., and Patterson, M. C. (2012). Dysphagia as a Risk Factor for Mortality in Niemann-Pick Disease Type C: Systematic Literature Review and Evidence from Studies with Miglustat. Orphanet J. Rare Dis. 7, 76. doi:10.1186/1750-1172-7-76

Wei, H., Qin, Z.-H., Senatorov, V. V., Wei, W., Wang, Y., Qian, Y., et al. (2001). Lithium Suppresses Excitotoxicity-Induced Striatal Lesions in a Rat Model of Huntington's Disease. Neuroscience 106, 603-612. doi:10.1016/s0306-4522(01) 00311-6

Wraith, J. E., Guffon, N., Rohrbach, M., Hwu, W. L., Korenke, G. C., Bembi, B., et al. (2009). Natural History of Niemann-Pick Disease Type C in a Multicentre Observational Retrospective Cohort Study. Mol. Genet. Metab. 98, 250-254. doi:10.1016/j.ymgme.2009.06.009

Wraith, J. E., Vecchio, D., Jacklin, E., Abel, L., Chadha-Boreham, H., Luzy, C., et al. (2010). Miglustat in Adult and Juvenile Patients with Niemann-Pick Disease Type C: Long-Term Data from a Clinical Trial. Mol. Genet. Metab. 99, 351-357. doi:10.1016/j.ymgme.2009.12.006

Xie, C., Gong, X.-M., Luo, J., Li, B.-L., and Song, B.-L. (2017). AAV9-NPC1 Significantly Ameliorates Purkinje Cell Death and Behavioral Abnormalities in Mouse NPC Disease. J. Lipid Res. 58, 512-518. doi:10.1194/jlr.M071274

Yanjanin, N. M., Vélez, J. I., Gropman, A., King, K., Bianconi, S. E., Conley, S. K., et al. (2009). Linear Clinical Progression, Independent of Age of Onset, in Niemann-Pick Disease, Type C. Am. J. Med. Genet. 9999B, a-n. doi:10.1002/ ajmg.b.30969

Youdim, M. B., and Arraf, Z. (2004). Prevention of MPTP (N-Methyl-4-Phenyl1,2,3,6-Tetrahydropyridine) Dopaminergic Neurotoxicity in Mice by Chronic Lithium: Involvements of Bcl-2 and Bax. Neuropharmacology 46, 1130-1140. doi:10.1016/j.neuropharm.2004.02.005

Zhang, H., Wang, Y., Lin, N., Yang, R., Qiu, W., Han, L., et al. (2014). Diagnosis of Niemann-Pick Disease Type C with 7-ketocholesterol Screening Followed by NPC1/NPC2 Gene Mutation Confirmation in Chinese Patients. Orphanet J. Rare Dis. 9, 82. doi:10.1186/1750-1172-9-82

Zhang, X., Heng, X., Li, T., Li, L., Yang, D., Zhang, X., et al. (2011). Long-Term Treatment with Lithium Alleviates Memory Deficits and Reduces Amyloid- $\beta$ Production in an Aged Alzheimer's Disease Transgenic Mouse Model. Jad 24, 739-749. doi:10.3233/JAD-2011-101875

Conflict of Interest: The authors declare that the research was conducted in the absence of any commercial or financial relationships that could be construed as a potential conflict of interest.

Copyright (c) 2021 Han, Zhang, Yi, Liu, Maegawa, Zou, Wang, Wu and Ye. This is an open-access article distributed under the terms of the Creative Commons Attribution License (CC BY). The use, distribution or reproduction in other forums is permitted, provided the original author(s) and the copyright owner(s) are credited and that the original publication in this journal is cited, in accordance with accepted academic practice. No use, distribution or reproduction is permitted which does not comply with these terms. 